\title{
Modülüs Fonksiyonu ile Tanımlanmış Genelleştirilmiş Büyük Lebesgue Dizi Uzaylarının Topolojik Bazı Özellikleri
}

\author{
Some Topological Properties of Generalized Grand Lebesgue Sequence Spaces Defined by \\ Modulus Function
}

\author{
Oğuz OĞUR*1,a \\ ${ }^{1}$ Giresun Üniversitesi, Fen-Edebiyat Fakültesi, Matematik Bölümü, 28200, Giresun
}

• Geliş tarihi / Received: 04.05.2020 • Düzeltilerek geliş tarihi / Received in revised form: 08.09.2020 • Kabul tarihi / Accepted: 25.09 .2020

$\ddot{O} z$

Bu çalışmada, Rafeiro vd. (2018) tarafından tanımlanan büyük Lebesgue dizi uzayları modülüs fonksiyonu yardımıyla genelleştirildi. Ayrıca, bu uzayların bazı topolojik ve kapsama özellikleri incelendi.

Anahtar kelimeler: Genelleştirilmiş Büyük Lebesgue Dizi Uzayları, Modülüs Fonksiyonu, Paranormlu Uzaylar.

\begin{abstract}
In this paper, the grand Lebesgue sequence spaces defined by Rafeiro vd. (2018) were generalized using modulus function. Also, some topological and inclusion properties of these spaces were examined.
\end{abstract}

Keywords: Generalized Grand Lebesgue Sequence Spaces, Modulus Function, Paranormed Spaces.

*a Oğuz OĞUR; oguz.ogur@giresun.edu.tr, Tel: (0454) 31010 00, orcid.org/0000-0002-3206-5330 


\section{Giriş}

$\mathrm{Bu}$ çalışmada, $\mathbb{N}, \mathbb{R}$ ve $\mathbb{C}$ sembolleri ile sırasıyla doğal sayılar, reel sayılar ve kompleks sayılar kümeleri gösterilsin. Modülüs fonksiyon kavramı ilk defa Nakano (1953) tarafından tanımlanmış ve temel bazı özellikleri verilmiştir. Aşağıdaki dört koşulu sağlayan $f:[0, \infty) \rightarrow[0, \infty)$ fonksiyonuna bir modülüs fonksiyonu denir:

1) $f(x)=0 \Leftrightarrow x=0$,

2) her $x, y \in[0, \infty)$ için $f(x+y) \leq f(x)+f(y)$,

3) $f$ fonksiyonu artandır,

4) Sifırın sağında $f$ fonksiyonu süreklidir.

Böylece modülüs fonksiyonun $[0, \infty)$ aralığında sürekli olduğu sonucu çıkarılır. Modülüs fonksyonları sınırlı veya sınırsız olabilirler. Örneğin, $f(x)=\frac{x}{x+1}$ fonksiyonu sınırlı fakat $0<p<1$ için $f(x)=x^{p}$ sınırsızdır. Modülüs fonksiyonunun tanımındaki 2) özelliğinden her $n \in \mathbb{N}$ için $f(n x) \leq n f(x)$ sağlanır. Buradan her $n \in \mathbb{N}$ için

$f(x) \leq f\left(n x \frac{1}{n}\right) \leq n f\left(\frac{x}{n}\right)$

ve böylece

$\frac{1}{n} f(x) \leq f\left(\frac{x}{n}\right)$

eşitsizliği elde edilir. $f$ bir modülüs fonksiyonu ve $w$ kompleks dizilerin uzayı olmak üzere

$L(f)=\left\{x \in w: \sum_{k=1}^{\infty} f\left(\left|x_{k}\right|\right)<\infty\right\}$

uzayı bir FK-uzayıdır (Ruckle, 1973). Ruckle, bu uzayı A. Wilansky” nin “ $\left\{e_{1}, e_{2}, \ldots\right\}$ birim vektörlerinin sınırlı kümesini bulunduran en küçük FK uzayı var mıdır?" sorusunun cevabını ararken tanımlamıştır. Yine, bütün $L(f)$ uzaylarının arakesitinin sonlu bütün dizilerin uzayı olduğunu göstermiştir. Eğer pozitif $x$ reel sayıları için $f(x)=x$ alınırsa $\mathrm{L}(f)$ uzayı $\ell_{1}$ uzayına indirgenir. Bu uzaylar birçok yazar tarafından çalışılmıştır (Maddox, 1986; Savaş, 1999; Malkowsky ve Savaş, 2000; Oğur, 2015). Ayrıca, Oğur ve Duyar (2016) modülüs fonksiyonu yardımıyla Lorentz dizi uzaylarını genelleştirmiştir.

$X$ bir vektör uzayı olmak üzere $p: X \rightarrow[0, \infty)$ fonksiyonuna aşağıdaki koşulları sağlaması durumunda bir paranorm denir;

i) $\quad p(0)=0$,

ii) her $x \in X$ için $p(-x)=p(x)$,

iii) $\quad x \in X, \lambda \in \mathbb{C},\left(\lambda_{n}\right), \mathbb{C}$ uzayında bir dizi ve $\left(x_{n}\right), X$ vektör uzayında bir dizi olmak üzere

eğer $n \rightarrow \infty$ iken $\left|\lambda_{n}-\lambda\right| \rightarrow 0$ ve $p\left(x_{n}-x\right) \rightarrow 0$ ise $p\left(\lambda_{n} x_{n}-\lambda x\right) \rightarrow 0 \quad$ sağlanır. ( $\mathrm{Bu}$ özelliğe skalerle çarpımın sürekliliği denir).

$1<p<\infty$ olmak üzere $L^{p)}$ büyük Lebesgue uzayı ilk olarak Iwanec ve Sbordone (1992) tarafindan tanımlanmıştır. Jain ve Kumari (2012) bu uzayları $\Lambda_{q), w}, 0<q<\infty$, büyük Lorentz uzaylarına taşımışlardır. Samko ve Umarkhadzhiev (2018) bu uzayların bazı temel özelliklerini çalışmışlardır. Rafeiro vd (2018) $\ell^{p), \theta}, 1 \leq p<\infty$ ve $\theta>0$, büyük Lebesgue dizi uzaylarını tanımlamış ve bu uzaylar üzerinde tanımlı bazı operatörlerin özelliklerini çalışmışlardır. Bu çalışmada, Rafeiro vd (2018) tarafından tanımlanan $\ell^{p), \theta}$ uzayı $f$ modülüs fonksiyonu yardımıyla genelleştirilmiş ve bazı temel özellikleri gösterilmiştir. $f$ modülüs fonksiyonu verilsin. $1 \leq p<\infty$ ve $\theta>0$ için

$\ell_{p), \theta}(f)=\left\{x \in w: \sup _{\varepsilon>0}\left(\varepsilon^{\theta} \sum_{k=1}^{\infty}\left[f\left(\left|x_{k}\right|\right)\right]^{p(1+\varepsilon)}\right)^{\frac{1}{p(1+\varepsilon)}}<\infty\right\}$

kümesi tanımlansın. 
$\ell_{p), \theta}(f)$ için geçerli bütün özellikler, $f(x)=x$ alındığında $\ell^{p), \theta}$ büyük Lebesgue dizi uzayı elde edileceğinden, bu uzaylar üzerinde çalışmak daha avantajlı olacaktır.

\section{Bulgular}

Bu bölümde, $\ell_{p), \theta}(f)$ 'nin bazı topolojik ve kapsama özellikleri verilmiştir.

Teorem 2.1: $f$ modülüs fonksiyonu olmak üzere $\ell_{p), \theta}(f), 1 \leq p<\infty$, kompleks sayılar cismi üzerinde bir vektör uzayıdır.

İspat: $\ell_{p), \theta}(f)^{\prime}$ nin $w$ uzayının bir alt uzayı olduğunu göstermek yeterlidir. Keyfi $a=\left(a_{k}\right), b=\left(b_{k}\right) \in$ $\ell_{p), \theta}(f)$ için

$$
\begin{aligned}
\sup _{\varepsilon>0}\left(\varepsilon^{\theta} \sum_{k=1}^{\infty}\left[f\left(\left|a_{k}+b_{k}\right|\right)\right]^{p(1+\varepsilon)}\right)^{\frac{1}{p(1+\varepsilon)}} & \leq \sup _{\varepsilon>0}\left(\varepsilon^{\theta} \sum_{k=1}^{\infty}\left[f\left(\left|a_{k}\right|+\left|b_{k}\right|\right)\right]^{p(1+\varepsilon)}\right)^{\frac{1}{p(1+\varepsilon)}} \\
& \leq \sup _{\varepsilon>0}\left(\varepsilon^{\theta} \sum_{k=1}^{\infty}\left[f\left(\left|a_{k}\right|\right)+f\left(\left|b_{k}\right|\right)\right]^{p(1+\varepsilon)}\right)^{\frac{1}{p(1+\varepsilon)}} \\
& \leq \sup _{\varepsilon>0}\left(\varepsilon^{\theta} \sum_{k=1}^{\infty}\left[f\left(\left|a_{k}\right|\right)\right]^{p(1+\varepsilon)}\right)^{\frac{1}{p(1+\varepsilon)}}+ \\
+ & \sup _{\varepsilon>0}\left(\varepsilon^{\theta} \sum_{k=1}^{\infty}\left[f\left(\left|a_{k}\right|\right)\right]^{p(1+\varepsilon)}\right)^{\frac{1}{p(1+\varepsilon)}} \\
& <\infty .
\end{aligned}
$$

Keyfi $\mu \in \mathbb{C}$ için $|\mu| \leq T_{\mu}$ olacak şekilde bir $T_{\mu} \in \mathbb{N}$ vardır. Buradan, modülüs fonksiyonunun özelliğinden

$$
\begin{aligned}
\sup _{\varepsilon>0}\left(\varepsilon^{\theta} \sum_{k=1}^{\infty}\left[f\left(\left|\mu a_{k}\right|\right)\right]^{p(1+\varepsilon)}\right)^{\frac{1}{p(1+\varepsilon)}} & \leq \sup _{\varepsilon>0}\left(\varepsilon^{\theta} \sum_{k=1}^{\infty}\left[f\left(\left|T_{\mu} a_{k}\right|\right)\right]^{p(1+\varepsilon)}\right)^{\frac{1}{p(1+\varepsilon)}} \\
& \leq T_{\mu} \sup _{\varepsilon>0}\left(\varepsilon^{\theta} \sum_{k=1}^{\infty}\left[f\left(\left|a_{k}\right|\right)\right]^{p(1+\varepsilon)}\right)^{\frac{1}{p(1+\varepsilon)}} \\
& <\infty
\end{aligned}
$$

bulunur. Bu ise $\ell_{p), \theta}(f)^{\prime}$ nin bir kompleks vektör uzayı olduğunu gösterir.

Teorem 2.2: $1 \leq p<\infty$ olmak üzere $\ell_{p), \theta}(f)$ uzay1

$g_{\theta, f}(a)=\sup _{\varepsilon>0}\left(\varepsilon^{\theta} \sum_{k=1}^{\infty}\left[f\left(\left|a_{k}\right|\right)\right]^{p(1+\varepsilon)}\right)^{\frac{1}{p(1+\varepsilon)}}$

fonksiyonu ile bir paranormlu uzaydır.

İspat: $g_{\theta, f}$ fonksiyonunun tanımından $g_{\theta, f}(a)=g_{\theta, f}(-a)$ ve $g_{\theta, f}(0)=0$ olduğu kolayca görülür. Ayrıca, (5) denkleminden $g_{\theta, f}(a+b) \leq g_{\theta, f}(a)+g_{\theta, f}(b)$ sağlanır. Eğer $g_{\theta, f}$ fonksiyonunun skalerle çarpımın sürekliliğini sağladığı gösterilirse ispat tamamlanır. Bunun için $m \rightarrow \infty$ iken $g_{\theta, f}\left(a^{(m)}-a\right) \rightarrow$ 0 ve $\left|\lambda^{(m)}-\lambda\right| \rightarrow 0$ özelliklerini sağlayan $\left(a^{(m)}\right) \subset \ell_{p), \theta}(f)$ ve $\left(\lambda^{(m)}\right) \subset \mathbb{C}$ dizileri verilsin. $g_{\theta, f}$ fonksiyonunun üçgen eşitsizliği özelliğinden

$g_{\theta, f}\left(\lambda^{(m)} a^{(m)}-\lambda a\right) \leq g_{\theta, f}\left(\lambda^{(m)} a^{(m)}-\lambda^{(m)} a\right)+g_{\theta, f}\left(\lambda^{(m)} a-\lambda a\right)$

bulunur. $R=\left(\llbracket \sup _{m}\left|\lambda^{(m)}\right| \rrbracket+1\right)$ olmak üzere modülüs fonksiyonunun monotonluğu kullanılırsa 


$$
\begin{aligned}
g_{\theta, f}\left(\lambda^{(m)} a^{(m)}-\lambda^{(m)} a\right) & =\sup _{\varepsilon>0}\left(\varepsilon^{\theta} \sum_{k=1}^{\infty}\left[f\left(\left|\lambda^{(m)} a_{k}^{(m)}-\lambda^{(m)} a_{k}\right|\right)\right]^{p(1+\varepsilon)}\right)^{\frac{1}{p(1+\varepsilon)}} \\
& =\sup _{\varepsilon>0}\left(\varepsilon^{\theta} \sum_{k=1}^{\infty}\left[f\left(\left|\lambda^{(m)}\right|\left|a_{k}^{(m)}-a_{k}\right|\right)\right]^{p(1+\varepsilon)}\right)^{\frac{1}{p(1+\varepsilon)}} \\
& \leq R \sup _{\varepsilon>0}\left(\varepsilon^{\theta} \sum_{k=1}^{\infty}\left[f\left(\left|a_{k}^{(m)}-a_{k}\right|\right)\right]^{p(1+\varepsilon)}\right)^{\frac{1}{p(1+\varepsilon)}} \\
& =R g_{\theta, f}\left(a^{(m)}-a\right)
\end{aligned}
$$

bulunur. Buradan $m \rightarrow \infty$ iken

$$
g_{\theta, f}\left(\lambda^{(m)} a^{(m)}-\lambda^{(m)} a\right) \rightarrow 0
$$

olduğu görülür. $m \rightarrow \infty$ iken $\left|\lambda^{(m)}-\lambda\right| \rightarrow 0$ olduğundan her $m \in \mathbb{N}$ için

$\left|\lambda^{(m)}-\lambda\right| \leq Q$

olacak şekilde bir $Q$ doğal sayısı bulunabilir. Keyfi pozitif $\delta$ sayısı verilsin. $a \in \ell_{p), \theta}(f)$ olduğundan öyle bir $k_{0} \in \mathbb{N}$ vardır öyleki

$$
\begin{aligned}
\sup _{\varepsilon>0}\left(\varepsilon^{\theta} \sum_{k=k_{0}}^{\infty}\left[f\left(\left|\lambda^{(m)}-\lambda\right|\left|a_{k}\right|\right)\right]^{p(1+\varepsilon)}\right)^{\frac{1}{p(1+\varepsilon)}} & \leq \sup _{\varepsilon>0}\left(\varepsilon^{\theta} \sum_{k=k_{0}}^{\infty}\left[f\left(Q\left|a_{k}\right|\right)\right]^{p(1+\varepsilon)}\right)^{\frac{1}{p(1+\varepsilon)}} \\
& \leq Q \sup _{\varepsilon>0}\left(\varepsilon^{\theta} \sum_{k=k_{0}}^{\infty}\left[f\left(\left|a_{k}\right|\right)\right]^{p(1+\varepsilon)}\right)^{\frac{1}{p(1+\varepsilon)}} \\
& <\frac{\delta}{2}
\end{aligned}
$$

olur. Buradan her $m \in \mathbb{N}$ için

$\sup _{\varepsilon>0}\left(\varepsilon^{\theta} \sum_{k=k_{0}}^{\infty}\left[f\left(\left|\lambda^{(m)} a_{k}-\lambda a_{k}\right|\right)\right]^{p(1+\varepsilon)}\right)^{\frac{1}{p(1+\varepsilon)}}<\frac{\delta}{2}$

olduğu elde edilir. Yine $f$ modülüs fonksiyonunun sürekliliğinden $m \rightarrow \infty$ iken

$\sup _{\varepsilon>0}\left(\varepsilon^{\theta} \sum_{k=1}^{k_{0}-1}\left[f\left(\left|\lambda^{(m)} a_{k}-\lambda a_{k}\right|\right)\right]^{p(1+\varepsilon)}\right)^{\frac{1}{p(1+\varepsilon)}}<\frac{\delta}{2}$

bulunur. Buradan, (13) ve (14) ifadeleri kullanılırsa $m \rightarrow \infty$ iken

$g_{\theta, f}\left(\lambda^{(m)} a-\lambda a\right) \rightarrow 0$

olur. Böylece, (10) ve (15) ifadelerinden $m \rightarrow \infty$ iken

$g_{\theta, f}\left(\lambda^{(m)} a^{(m)}-\lambda a\right) \rightarrow 0$

elde edilir. Bu ise $\ell_{p), \theta}(f)$ uzayının $g_{\theta, f}$ fonksiyonu ile birlikte bir paranormlu uzay olduğunu gösterir.

Uyarı 2.1: (7) ifadesiyle verilen $g_{\theta, f}$ fonksiyonunun tanımdaki supremumun $\varepsilon$ ' nun sonlu bir aralı̆̆ında alınması yeterlidir. $x>0$ için $x \rightarrow x e^{x}$ fonksiyonunun ters fonksiyonu $W(x)$ (Lambert fonksiyonu) olmak üzere 
$g_{\theta, f}(a)=\sup _{0<\varepsilon<\frac{1}{W(1 / e)}}\left(\varepsilon^{\theta} \sum_{k=1}^{\infty}\left[f\left(\left|a_{k}\right|\right)\right]^{p(1+\varepsilon)}\right)^{\frac{1}{p(1+\varepsilon)}}$

olur. Burada $W(1 / e) \cong 3,57$ şeklindedir (Rafeiro vd, 2018).

Teorem 2.3: $1 \leq p<\infty$ olmak üzere $\ell_{p), \theta}(f)$ uzayı $g_{\theta, f}(a)$ paranormu ile bir tam uzaydır.

İspat: $\left(a^{(s)}\right), \ell_{p), \theta}(f)$ uzayında keyfi bir Cauchy dizisi olsun. Bu takdirde, her $\tau>0$ için $s, t \geq n_{0}$ olduğunda

$$
\begin{aligned}
g_{\theta, f}\left(a^{(s)}-a^{(t)}\right) & =\sup _{0<\varepsilon<\frac{1}{W(1 / e)}}\left(\varepsilon^{\theta} \sum_{m=1}^{\infty}\left[f\left(\left|a_{m}^{(s)}-a_{m}^{(t)}\right|\right)\right]^{p(1+\varepsilon)}\right)^{\frac{1}{p(1+\varepsilon)}} \\
& <f(\tau)\left(\frac{1}{W(1 / e)}\right)^{\frac{\theta}{p\left(1+\frac{1}{W(1 / e)}\right)}}
\end{aligned}
$$

olacak şekilde $n_{0}$ doğal sayısı vardır. Buradan, $s, t \geq n_{0}$ olduğunda

$\left|a_{m}^{(s)}-a_{m}^{(t)}\right|<\tau$

olur. Bu ise her $m$ doğal sayısı için $\left(a_{m}^{(s)}\right)$ dizisinin $\mathbb{C}$ uzayında bir Cauchy dizisi olduğunu gösterir. Buradan, $s \rightarrow \infty$ iken

$a_{m}^{(s)} \rightarrow a_{m}$

olacak şekilde $a_{m} \in \mathbb{C}$ vardır. $a=\left(a_{m}\right)$ olarak tanımlansın. $a=a^{(s)}+\left(a^{(s)}-a\right)$ olduğundan $a \in$ $\ell_{p), \theta}(f)$ olur. Ayrica, $s, t \rightarrow \infty$ iken

$g_{\theta, f}\left(a^{(s)}-a\right) \leq g_{\theta, f}\left(a^{(s)}-a^{(t)}\right)+g_{\theta, f}\left(a^{(t)}-a\right) \rightarrow 0$

bulunur. $\mathrm{Bu} \ell_{p), \theta}(f)$ uzayının üzerindeki paranorm ile bir tam uzay olduğunu gösterir.

Teorem 2.4: $f$ ve $h$ iki modülüs fonksiyonu verilsin. Aşağıdaki kapsamalar sağlanır;

i) $\quad$ Ĕger $\limsup \frac{f(t)}{h(t)}<\infty$ ise $\ell_{p), \theta}(h) \subset \ell_{p), \theta}(f)$.

ii) $1 \leq p<\infty$ için $\ell_{p), \theta}(h) \cap \ell_{p), \theta}(f) \subset \ell_{p), \theta}(f+h)$.

İspat:

i) Hipotezden, her $t$ pozitif reel sayısı için

$f(t) \leq K h(t)$

olacak şekilde $K>0$ sayısı vardır. Böylece, $x \in \ell_{p), \theta}(h)$ için

$f\left(x_{k}\right) \leq K h\left(x_{k}\right)$

yaz1lır. Buradan $\sup _{0<\varepsilon<\frac{1}{W(1 / e)}}\left(\varepsilon^{\theta} \sum_{k=1}^{\infty}\left[f\left(\left|x_{k}\right|\right)\right]^{p(1+\varepsilon)}\right)^{\frac{1}{p(1+\varepsilon)}} \leq K g_{\theta, h}(x)<\infty$

bulunur. Bu ise $x \in \ell_{p), \theta}(f)$ olduğunu gösterir. 
ii) $x \in \ell_{p), \theta}(h) \cap \ell_{p), \theta}(f)$ verilsin. Buradan,

$$
\begin{aligned}
\sup _{0<\varepsilon<\frac{1}{W(1 / e)}}\left(\varepsilon^{\theta} \sum_{k=1}^{\infty}\left[(f+h)\left(\left|x_{k}\right|\right)\right]^{p(1+\varepsilon)}\right)^{\frac{1}{p(1+\varepsilon)}} & \leq \sup _{0<\varepsilon<\frac{1}{W(1 / e)}}\left(\varepsilon^{\theta} \sum_{k=1}^{\infty}\left[f\left(\left|x_{k}\right|\right)\right]^{p(1+\varepsilon)}\right)^{\frac{1}{p(1+\varepsilon)}} \\
+ & \sup _{0<\varepsilon<\frac{1}{W(1 / e)}}\left(\varepsilon^{\theta} \sum_{k=1}^{\infty}\left[h\left(\left|x_{k}\right|\right)\right]^{p(1+\varepsilon)}\right)^{\frac{1}{p(1+\varepsilon)}} \\
& \leq g_{\theta, f}(x)+g_{\theta, h}(x) \\
& <\infty
\end{aligned}
$$

elde edilir. Bu ise $x \in \ell_{p), \theta}(f+h)$ olduğunu gösterir.

\section{Tartışma ve Sonuçlar}

$\mathrm{Bu}$ çalışmada, $1 \leq p<\infty$ olmak üzere büyük Lebesgue dizi uzayları modülüs fonksiyonu yardımıyla $\ell_{p), \theta}(f)$ uzayına genelleştirilmiş ve bu uzayın temel topolojik özellikleri incelenmiştir. Ayrıca, bazı kapsama özellikleri verilmiştir.

\section{Kaynaklar}

Iwaniec, T. ve Sbordone, C., 1992. On the Integrability of the Jacobian Under Minimal Hypotheses. Archive for Rational Mechanics and Analysis. 119(2), 129-143.

Jain, P. ve Kumari, S., 2012. On Grand Lorentz Spaces and the Maximal Operator. Georgian Mathematical Journal, 19, 235-246.

Maddox, I.J., 1986. Sequence Spaces Defined by a Modulus. Mathematical Proceeding of the Cambridge Philosophical Society, 100, 161-166.

Malkowsky, E. ve Savaş, E., 2000. Some $\lambda$-Sequence Spaces Defined by a Modulus. Archiv der Mathematik, 36(3), 219-228.

Nakano, H., 1953. Concave Modular. Journal of the Mathematical Society of Japan, 5, 29-49.

Oğur, O., 2015. A New Double Cesaro Sequence Space Defined by Modulus Functions. Journal of Applied Functional Analysis, 10(1), 109-116.

Oğur, O. ve Duyar, C., 2016. On Generalized Lorentz Sequence Space Defined by Modulus Functions. Filomat, 30(2), 497-504.

Rafeiro, H., Samko, S., Umarkhadzhiev S., 2018. Grand Lebesgue Sequence Spaces. Georgian Mathematical Journal, 19(2), 235-246.

Ruckle, W. H.,1973. FK-Spaces in which the Sequence of Coordinate Vectors is Bounded. Canadian Journal of Mathematics, 25, 973-978.
Samko, S. ve Umarkhadzhiev S., 2017. On Grand Lebesgue Spaces on Sets of Infinite Measure. Mathematische Nachrichten, 290, 913-919.

Savaş, E., 1999. On Some Generalized Sequence Spaces Defined by a Modulus. Indian Journal of Pure and Applied Mathematics, 30(5), 459-464.

Wilansky, A., 1964. Functional Analysis: New York, Blaisdell. 